\title{
High expression of elF3d is associated with poor prognosis in patients with gastric cancer
}

\author{
This article was published in the following Dove Press journal: \\ Cancer Management and Research \\ 25 October 2017 \\ Number of times this article has been viewed
}

\author{
Jiaqi $\mathrm{He}^{\prime}$ \\ Xuefei Wang ${ }^{2}$ \\ Jianhua Cai' \\ Wei Wang' \\ Xinyu Qin ${ }^{2}$ \\ 'Department of General Surgery, \\ Huadong Hospital, ${ }^{2}$ Department of \\ General Surgery, Zhongshan Hospital, \\ Fudan University, Shanghai, People's \\ Republic of China
}

Correspondence: Wei Wang Department of General Surgery, Huadong Hospital, Fudan University, 22I, West Yan'an Road, Shanghai, 200040, People's Republic of China

Email hd_weiwang@I63.com

Xinyu Qin

Department of General Surgery, Zhongshan Hospital, Fudan University, 180, Fenglin Road, Shanghai, 200032,

People's Republic of China

Email zs_qinxinyu@163.com
Background: Eukaryotic initiation factor 3 subunit d (eIF3d) is the largest subunit of eIF3, which is shown to promote protein synthesis in cancer cells. Increased expression of eIF3d has been shown in some types of cancers, but has not been previously studied in gastric cancer (GC). Thus, the aim of this study was to analyze eIF3d expression in GC.

Patients and methods: Expression of eIF3d was detected by immunohistochemistry in GC tissues and adjacent noncancerous (ANC) tissues. Samples were obtained from 210 patients with GC who had received curative gastrectomy. Clinicopathological features and survival rate were also analyzed.

Results: Expression rates of eIF3d in GC and ANC were $45.2 \%$ and $21.0 \%$, respectively. High expression of eIF3d protein was significantly related to tumor stage, as determined by lymph node metastasis and depth of invasion $(p<0.05)$. The Kaplan-Meier survival curves showed that patients with high eIF3d expression had a significantly poor overall survival $(p=0.005)$. Multivariate Cox regression analyses showed that the level of eIF3d was an independent predictive factor of poor prognosis for $\mathrm{GC}(p=0.017)$.

Conclusion: Expression of eIF3d was upregulated in GC. High expression of eIF3d was determined as an independent poor prognostic factor in GC. It is suggested that eIF3d could be a good biomarker in GC.

Keywords: gastric cancer, eIF3d, eukaryotic translation initiation factors 3d, biomarker, prognosis

\section{Introduction}

Gastric cancer (GC) remains one of the leading causes of cancer-related mortality worldwide. ${ }^{1}$ Although great improvements have been made in the treatment of GC, fiveyear survival rates have remained very low, approximating $20 \%$, due to the tendency for early invasion and metastasis. ${ }^{2}$ Clinically, TNM stage is predominantly used to predict the outcome of GC patients. However, growing evidence has suggested that patients with the same stage might have vastly different prognoses due to the heterogeneity of tumors. ${ }^{3,4}$ Therefore, it is urgent to find useful biomarkers to refine risk stratification and survival prognosis.

Eukaryotic initiation factor 3 (eIF3) is a protein complex involved in the initiation pathway. Functional eIF3 binds to the $40 \mathrm{~S}$ ribosomal subunit and promotes the formation of the $40 \mathrm{~S}$ initiation complex. ${ }^{6,7}$ It has been shown that eIF3 is the largest initiation factor composed of 13 non-identical subunits denoted as eIF3a-m. ${ }^{5-7} \mathrm{Sev}-$ eral eIF3 subunits, such as eIF3a, eIF3b, eIF3c, eIF3h, eIF3i, and eIF3e, have been 
demonstrated to promote cell proliferation by initiating protein translation in cancers. ${ }^{8}$ In addition, eIF3f subunit has been shown to be downregulated in cancers. ${ }^{9}$ Recent studies have reported that deregulation of eIF3 subunits is implicated in tumorigenesis. ${ }^{10-12}$

The eIF3 subunit d (eIF3d), categorized as the largest subunit of eIF3, is crucial for the functional activity of eIF3. ${ }^{13,14}$ Recent studies have reported eIF3d over expression in several malignant tumors, including prostate cancer, ${ }^{15}$ colon cancer, ${ }^{16}$ and melanoma. ${ }^{17}$ The eIF3d has also been identified as a potential therapeutic target in several cancer types. ${ }^{17-19}$ Similarly, Kim et al identified eIF3d as a predictive gene in GC. This information might be useful in establishing resistance against a combined treatment of cisplatin and fluorouracil. ${ }^{20}$

The expression of eIF3d in human GC tissue and its clinical significance have not been reported in the literature. In this study, we analyzed eIF3d expression in primary GC from 210 Chinese patients by immunohistochemical analysis and investigated the relationship of eIF3d and various clinicopathological factors.

\section{Patients and methods}

\section{Specimen source and patient information}

A total of 210 patients with $\mathrm{GC}$ who had received curative gastrectomy in the Department of General Surgery, Zhongshan Hospital, Fudan University, Shanghai, China, between January 2006 and October 2010 were enrolled in this study. Patients, age ranged from 22 to 87 years (mean age, 59.6 years). The mean follow-up period was 40.96 months with a range from 2 to 89 months. The TNM stage of tumors was evaluated according to the 7 th edition of the TNM Classification of GC. ${ }^{22}$ No patient underwent radiotherapy or chemotherapy before surgery. The $210 \mathrm{GC}$ tissues and 195 adjacent noncancerous (ANC) tissues were obtained from these specimens for immunohistochemical staining. All these specimens were fixed with $10 \%$ formaldehyde and embedded with paraffin, and were examined by a pathologist to confirm malignancy. The above cases were approved by the ethics committee of Huadong Hospital and informed consent forms were signed.

\section{Immunostaining analysis}

A polyclonal antibody against eIF3d (ab155419; Abcam, Cambridge, MA, USA) was used in this study. Specimens were sectioned into 3- to 4- $\mu \mathrm{m}$ slices. After routine xylene dewaxing and gradient ethanol hydration, the slides were blocked with 3\% hydrogen peroxide for $10 \mathrm{~min}$. After using the microwave antigen repair method, the slides were incubated with the anti-eIF3d Ab (diluted 1:40 in phosphate buffered saline $[\mathrm{PBS}]$ ) at $4^{\circ} \mathrm{C}$ for $24 \mathrm{~h}$. Sections were washed thrice with PBS, followed by the addition of diaminobenzidine for $6 \mathrm{~min}$. Slides were independently evaluated by two pathologists who were blinded to clinical data. The level of eIF3d was scored not only by staining intensity but also by the percentage of cells that exhibit eIF3d. The staining intensity was scored as 0 (no staining), 1 (weak), 2 (moderate), or 3 (intense staining). The percentage of positive cells was scored as follows: $0(\leq 5 \%), 1(6 \%$ to $25 \%), 2(26 \%$ to $50 \%)$, and $3(>50 \%)$. The total of the above two scores was graded as follows: 0 (score 0), 1 (score 1-2), 2 (score 3-4), and 3 (score 5-6), where the total scores of 0 or 1 were designated as low expression and 2 or 3 were designated as high expression. ${ }^{21}$

\section{Statistical analysis}

The SPSS 19 software package (IBM Corporation, Armonk, NY, USA) was used for statistical analysis. Student's $t$-test was used for continuous variables, whereas $\chi^{2}$ test or Fisher's exact test was used for proportions. The Kaplan-Meier method was used for survival analyses and the Cox regression model was used for prognostic factors, such as eIF3d and other clinicopathological features.

\section{Results Expression of elF3d in GC tissues}

The eIF3d staining results predominantly showed cytoplasmic staining in both the GC tissues and the ANC tissues (Figure 1). However, a sharp difference was found when eIF3d expression in GC was compared with that of ANC. The high eIF3d expression rate in GC tissues was 45.2\% (95/210) while that in ANC tissues was 21.0\% (41/195; $p<0.001)$.

\section{elF3d expression and clinicopathological features}

Expression of eIF3d (high expression in 95 patients; low expression in 115 patients) and clinicopathological features were analyzed. Table 1 summarizes the positive correlates of upregulated eIF3d expression with lymph node metastasis ( $p=0.041)$, tumor depth $(p=0.007)$, and late TNM stage (III/ IV) $(p=0.027)$. The data indicate that eIF3d did not correlate significantly with gender $(p=0.217)$, age $(p=0.724)$, primary tumor location $(p=0.403)$, or lymphovascular inva$\operatorname{sion}(p=0.312)$.

\section{elF3d as a predictor of overall survival in GC patients}

Kaplan-Meier survival curves showed that the cumulative survival rates were $75.6 \%$ ( 3 years) and $72.9 \%$ ( 5 years) in 

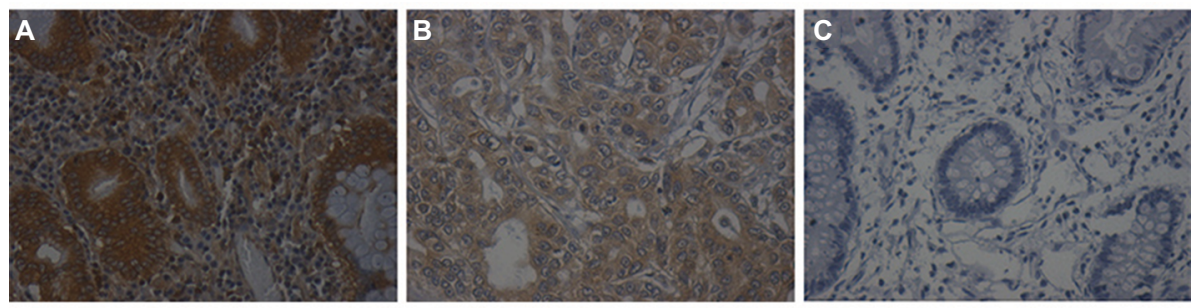

Figure I The expression of elF3d in gastric cancer tissues and adjacent noncancerous tissues.

Notes: (A) High elF3d expression in gastric cancer tissues. (B) Low elF3d expression in gastric cancer tissues. (C) Negative elF3d expression in adjacent noncancerous tissues. Magnification $\times 20$

Abbreviation: elF3d, eukaryotic initiation factor 3 subunit $d$.

Table I Relationship of elF3d expression with clinicopathological parameters of tumor

\begin{tabular}{|c|c|c|c|c|}
\hline \multirow[t]{2}{*}{ Clinical parameters } & \multirow[t]{2}{*}{$\mathbf{N}$} & \multicolumn{2}{|c|}{ elF3d expression } & \multirow[t]{2}{*}{$p$-value } \\
\hline & & Low (\%) & High (\%) & \\
\hline Total & 210 & $115(54.8)$ & $95(45.2)$ & \\
\hline Gender & & & & 0.239 \\
\hline Male & 135 & $78(57.8)$ & $57(42.2)$ & \\
\hline Female & 75 & $37(49.3)$ & $38(50.7)$ & \\
\hline Age, years & & & & 0.306 \\
\hline$<60$ & 109 & $56(5 I .4)$ & $53(48.6)$ & \\
\hline$\geq 60$ & 101 & $59(58.4)$ & $42(41.6)$ & \\
\hline Primary tumor location & & & & 0.403 \\
\hline Upper I/3 & 21 & II (52.4) & $10(47.6)$ & \\
\hline Middle $1 / 3$ & 49 & $29(59.2)$ & $20(40.8)$ & \\
\hline Lower $1 / 3$ & 130 & $72(55.4)$ & $58(44.6)$ & \\
\hline Multiple & 10 & $3(30.0)$ & $7(70.0)$ & \\
\hline Tumor differentiation & & & & 0.052 \\
\hline Well & 6 & I (20.0) & $5(80.0)$ & \\
\hline Moderate & 36 & $16(44.4)$ & $20(55.6)$ & \\
\hline Poor & 168 & $98(58.3)$ & 70 (4I.7) & \\
\hline Tumor depth & & & & $0.004 *$ \\
\hline $\mathrm{TI} / \mathrm{T} 2 / \mathrm{T} 3$ & 123 & $77(62.6)$ & $45(37.4)$ & \\
\hline T4 (serosa invasion) & 87 & $38(43.7)$ & $50(56.3)$ & \\
\hline Lymph node metastasis & & & & $0.04 I^{*}$ \\
\hline Negative & 73 & $47(64.4)$ & $26(35.6)$ & \\
\hline Positive & 137 & $68(49.6)$ & $69(50.4)$ & \\
\hline Distant metastasis & & & & 0.502 \\
\hline Negative & 205 & I I $3(55.1)$ & $92(44.9)$ & \\
\hline Positive & 5 & $2(40.0)$ & $3(60.0)$ & \\
\hline TNM stage & & & & 0.304 \\
\hline 1 & 65 & $41(63.1)$ & $24(26.9)$ & \\
\hline II & 44 & $25(56.8)$ & $19(43.2)$ & \\
\hline III & 96 & $47(49.0)$ & $49(51.0)$ & \\
\hline IV & 5 & $2(40.0)$ & $3(60.0)$ & \\
\hline Early or late stage & & & & $0.027^{*}$ \\
\hline $\mathrm{I} / \mathrm{II}$ & 106 & $66(62.3)$ & $40(37.7)$ & \\
\hline $\mathrm{III} / \mathrm{V}$ & 104 & $49(47.1)$ & $55(52.9)$ & \\
\hline Lymphovascular invasion & & & & 0.974 \\
\hline Negative & 166 & 91 (54.8) & $75(45.2)$ & \\
\hline Positive & 44 & $24(54.5)$ & $20(45.5)$ & \\
\hline
\end{tabular}

Note: ${ }^{*} p<0.05$ : statistical significance.

Abbreviation: elF3d, eukaryotic initiation factor 3 subunit $d$.

patients with lower eIF3d expression, and $59.5 \%$ (3 years) and $52.9 \%$ (5 years) in those with higher eIF3d expression. The overexpression of eIF3d was significantly correlated with poor overall survival of GC patients ( $p=0.005)$. The data were divided into subgroups according to the TNM stage. The 5-year survival rates were $97.6 \%$ and $95.8 \%$ in low and high eIF3d patients, respectively $(p=0.925)$ in stage I and $75.6 \%$ and $58.6 \%$, respectively $(p=0.274)$ in stage II. However, in stage III, the 5-year survival rate in low eIF3d patients was significantly higher than that of high eIF3d patients $(52.4 \%$ vs. $29.7 \%, p=0.046$; Figure 2). Furthermore, multivariate survival analysis was performed using the Cox multivariate regression model. The results revealed that eIF3d levels (HR=1.804; 95\% CI: 1.112-2.926; $p=0.017$ ), lymph nodes metastasis $(\mathrm{HR}=3.082 ; 95 \% \mathrm{CI}: 1.101-8.629 ; p=0.032)$, distant metastasis (HR=3.776; 95\% CI: $1.362-10.464 ; p=0.007$ ), lymphovascular invasion (HR=1.817; 95\% CI: 1.086-3.041; $p=0.023)$, and late TNM stage (HR $=2.886$; 95\% CI: 1.374 $6.509 ; p=0.005$ ) were statistically independent forecasting factors of adverse outcome for GC (Tables 2 and 3).

\section{Discussion}

GC is a highly heterogeneous disease with poor clinical outcomes. The traditional stage classification systems have limited capacity to predict the results of GC patients. More relevant studies should be undertaken to refine prognosis, comprehend progression, and improve treatment. Investigations have showed that protein synthesis is central to the occurrence and development of tumors. Translational control occurs in any phase of protein synthesis, and it is believed that the initiation phase of translation plays an important role during the process. ${ }^{23,24} \mathrm{eIF} 3$ is the largest and most intricate eIF, consisting of thirteen subunits. This polypeptide interacts with the 40S subunit of free-floating ribosomes and stabilizes the eIF2-GTP-Met-tRNAi complex. ${ }^{6,7}$ eIF3d is the largest subunit of eIF3 and its function may be crucial. Some studies showed that eIF3d was required for the stable association of eIF3 subunits. ${ }^{14}$ Based on these observations, it may be inferred that eIF3d is essential in promoting protein synthesis in the occurrence of cancer through certain molecular mechanisms. 
A

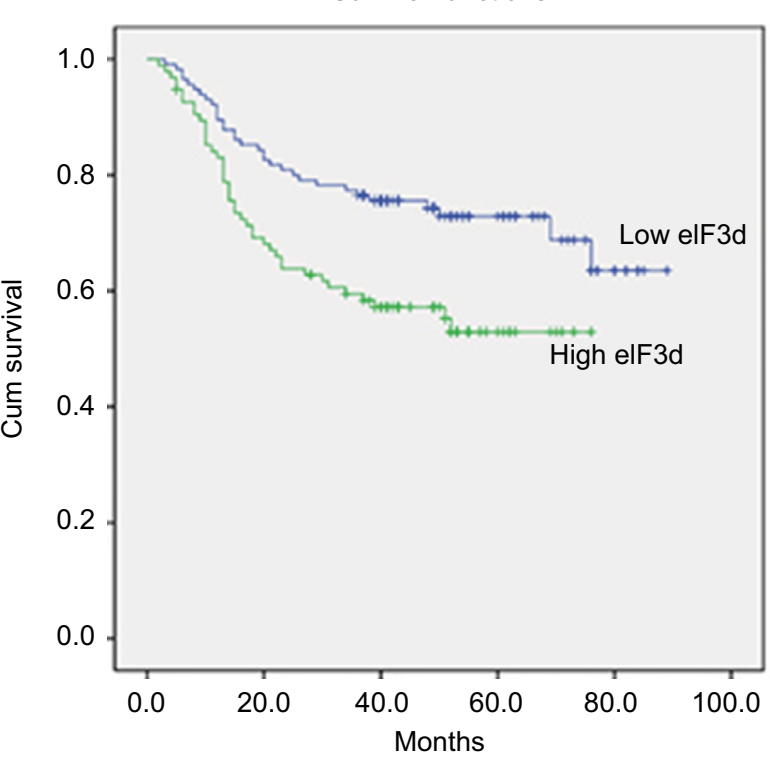

C

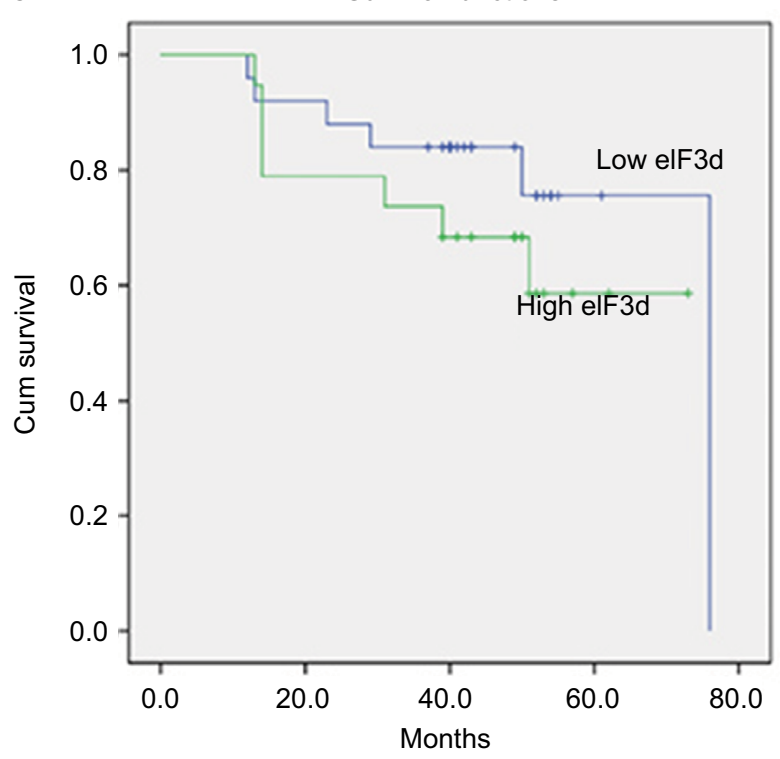

B

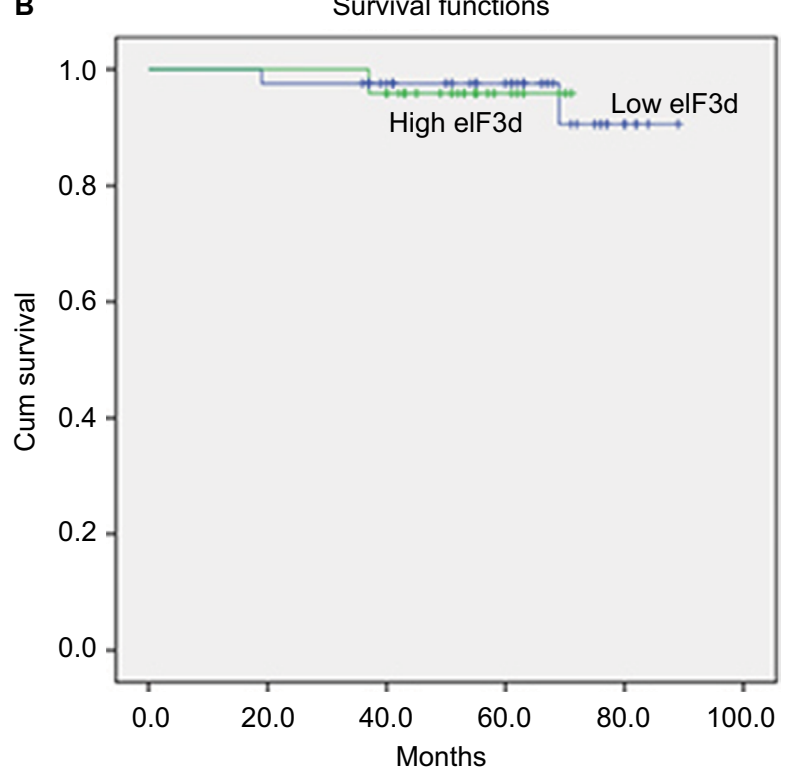

D

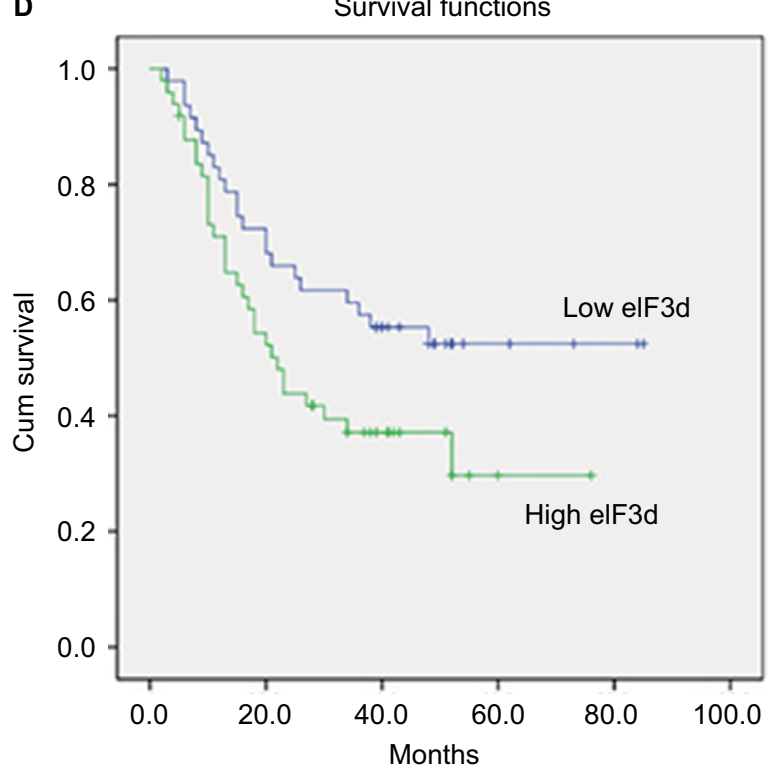

Figure 2 Kaplan-Meier analysis for overall survival of patients with gastric cancer according to elF3d expression.

Notes: (A) The overall survival in all patients. (B) The overall survival in patients with TNM I stage. (C) The overall survival in patients with TNM II stage. (D) The overall survival in patients with TNM III stage.

Abbreviations: Cum, cumulative; elF3d, eukaryotic initiation factor 3 subunit $d$.

Table 2 Univariate analysis of the correlation between clinicopathological parameters and survival time in patients with gastric cancer

\begin{tabular}{ll}
\hline Clinical parameters & p-value \\
\hline Age & 0.052 \\
Gender & 0.620 \\
Lymphovascular invasion & $<0.00 I^{*}$ \\
elF3d expression & $0.006^{*}$ \\
Tumor differentiation & 0.096 \\
Lymph node metastasis & $<0.00 I^{*}$ \\
Distant metastasis & $<0.00 I^{*}$ \\
Serosa invasion & $<0.00 I^{*}$ \\
\hline
\end{tabular}

Note: $*_{p}<0.05$ : statistical significance.

Abbreviation: elF3d, eukaryotic initiation factor 3 subunit $d$.
Previous studies have demonstrated that eIF3d was overexpressed in several human cancers, such as prostate cancer, ${ }^{15}$ colon cancer, ${ }^{16}$ and melanoma, ${ }^{17}$ while also resistant to chemotherapy. ${ }^{20}$ There have been recent studies on expression of other subunits of eIF3 in $\mathrm{GC}^{9,25}$; however, the role of eIF3d in GC remains unclear.

In this study, immunohistochemical staining was used to evaluate the level of eIF3d in GC and to analyze with the resultant clinicopathology and prognosis. The data showed that eIF3d was remarkably higher in GC tissues than companion ANC tissues. In addition, eIF3d was positively correlated with the tumor stage, as determined by lymph node 
Table 3 Multivariate analysis of the correlation between clinicopathological parameters and survival time in patients with gastric cancer

\begin{tabular}{|c|c|c|c|c|c|c|c|}
\hline Clinical parameters & B & SE & Wald & $d f$ & $p$-value & HR & $95 \% \mathrm{Cl}$ \\
\hline Age & 0.081 & 0.242 & 0.113 & I & 0.737 & 0.922 & $0.574-|.48|$ \\
\hline Gender & 0.029 & 0.264 & 0.012 & 1 & 0.911 & 0.971 & $0.579-1.627$ \\
\hline Lymphovascular invasion & 0.569 & 0.267 & 4.541 & 1 & $0.033^{*}$ & 0.566 & $0.336-0.955$ \\
\hline elF3d expression & 0.545 & 0.248 & 4.836 & 1 & $0.028^{*}$ & 0.580 & $0.357-0.942$ \\
\hline Tumor differentiation & 0.651 & 0.354 & 3.372 & 1 & 0.066 & 0.522 & $0.261-1.045$ \\
\hline Lymph node metastasis & 1.545 & 0.457 & II .442 & I & $0.00 I^{*}$ & 0.213 & $0.087-0.522$ \\
\hline Distant metastasis & 1.688 & 0.515 & $|0.73|$ & I & $0.001 *$ & 0.185 & $0.067-0.508$ \\
\hline Serosa invasion & 0.735 & 0.284 & 6.692 & I & $0.010 *$ & 0.480 & $0.275-0.837$ \\
\hline
\end{tabular}

Notes: B: Regression coefficient estimate, Wald: used for checking the B value. ${ }^{*} p<0.05$ : statistical significance.

Abbreviations: elF3d, eukaryotic initiation factor 3 subunit d; SE, Standard error; df, degrees of freedom; HR, hazard ratio; Cl, confidence interval.

metastasis and depth of invasion. The Kaplan-Meier survival analysis showed that patients with high eIF3d expression had significantly poorer survival compared with patients with low eIF3d expression, particularly those in stage III. This was consistent with the result that eIF3d overexpression was more frequent in late-stage patients. Furthermore, multivariate Cox regression analyses showed that the level of eIF3d was an independent predictive factor of poor prognosis for GC as well as lymph node metastasis, serosa invasion, and lymphovascular invasion. Thus, eIF3d might serve as an efficient prognostic biomarker in GC.

In consideration of its indispensable role in protein synthesis and cell proliferation, eIF $3 \mathrm{~d}$ could be a potential target for gene therapy in GC. It was reported that knockdown of eIF3d could inhibit the growth of colon and breast cancer cells by inducing cell-cycle arrest at the G2/M phase or suppressing the Wnt/ $\beta$-catenin signaling pathway. ${ }^{16,19}$ This study showed the relationship between eIF3d and the malignant behavior of $\mathrm{GC}$ at the immunohistochemical level implying that eIF3d may promote tumor growth. However, further studies should be performed at the cellular and molecular levels to reveal the underlying mechanism and should look for the possibility of making use of eIF3d in GC treatment.

\section{Conclusion}

To our knowledge, this study represents a first report identifying that eIF3d was upregulated in GC. Elevated expression of eIF3d was an independent poor prognostic factor in GC. We suggest that eIF3d could be a good biomarker in GC.

\section{Disclosure}

The authors report no conflicts of interest in this work.

\section{References}

1. Torre LA, Bray F, Siegel RL, Ferlay J, Lortet-Tieulent J, Jemal A. Global cancer statistics, 2012. CA Cancer J Clin. 2015;65(2):87-108.

2. Yoon H, Kim N. Diagnosis and management of high risk group for gastric cancer. Gut Liver. 2015;9(1):5-17.
3. Lim L, Michael M, Mann GB, Leong T. Adjuvant therapy in gastric cancer. J Clin Oncol. 2005;23(25):6220-6232.

4. Stock M, Otto F. Gene deregulation in gastric cancer. Gene. 2005;360(1):1-19.

5. Damoc E, Fraser CS, Zhou M, et al. Structural characterization of the human eukaryotic initiation factor 3 protein complex by mass spectrometry. Mol Cell Proteomics. 2007;6(7):1135-1146.

6. Jackson RJ, Hellen CU, Pestova TV. The mechanism of eukaryotic translation initiation and principles of its regulation. Nat Rev Mol Cell Biol. 2010;11(2):113-127.

7. Hershey JW. The role of eIF3 and its individual subunits in cancer. Biochim Biophys Acta. 2015;1849(7):792-800.

8. Dong Z, Zhang JT. Initiation factor eIF3 and regulation of mRNA translation, cell growth, and cancer. Crit Rev Oncol Hematol. 2006;59(3):169-180.

9. Li G, Wang N, Sun C, Li B. Decreased expression of eukaryotic initiation factor $3 \mathrm{f}$ is an adverse prognostic factor for stage I-III gastric cancer. World J Surg Oncol. 2014;12:72.

10. Zhang L, Pan X, Hershey JW. Individual overexpression of five subunits of human translation initiation factor eIF3 promotes malignant transformation of immortal fibroblast cells. J Biol Chem. 2007;282(8):5790-5800.

11. Wang Z, Chen J, Sun J, Cui Z, Wu H. RNA interference-mediated silencing of eukaryotic translation initiation factor 3, subunit B (EIF3B) gene expression inhibits proliferation of colon cancer cells. World $J$ Surg Oncol. 2012;10:119.

12. Dong Z, Liu LH, Han B, Pincheira R, Zhang JT. Role of eIF3 p170 in controlling synthesis of ribonucleotide reductase M2 and cell growth. Oncogene. 2004;23(21):3790-3801.

13. Villa N, Do A, Hershey JW, Fraser CS. Human eukaryotic initiation factor $4 \mathrm{G}(\mathrm{eIF} 4 \mathrm{G})$ protein binds to eIF $3 \mathrm{c}$, -d, and -e to promote mRNA recruitment to the ribosome. J Biol Chem. 2013;288(46):32932-32940.

14. Bandyopadhyay A, Lakshmanan V, Matsumoto T, Chang EC, Maitra U. Moe1 and spInt6, the fission yeast homologues of mammalian translation initiation factor 3 subunits p66 (eIF3d) and p48 (eIF3e), respectively, are required for stable association of eIF3 subunits. $J$ Biol Chem. 2002;277(3):2360-2367.

15. Gao Y, Teng J, Hong Y, et al. The oncogenic role of EIF3D is associated with increased cell cycle progression and motility in prostate cancer. Med Oncol. 2015;32(7):518.

16. Yu X, Zheng B, Chai R. Lentivirus-mediated knockdown of eukaryotic translation initiation factor 3 subunit $D$ inhibits proliferation of HCT116 colon cancer cells. Biosci Rep. 2014;34(6):e00161.

17. Sudo H, Tsuji AB, Sugyo A, et al. Knockdown of COPA, identified by loss-of-function screen, induces apoptosis and suppresses tumor growth in mesothelioma mouse model. Genomics. 2010;95(4):210-216.

18. Li H, Zhou F, Wang H, et al. Knockdown of EIF3D suppresses proliferation of human melanoma cells through $\mathrm{G} 2 / \mathrm{M}$ phase arrest. Biotechnol Appl Biochem. 2015;62(5):615-620.

19. Fan Y, Guo Y. Knockdown of eIF3D inhibits breast cancer cell proliferation and invasion through suppressing the Wnt/beta-catenin signaling pathway. Int J Clin Exp Pathol. 2015;8(9):10420-10427. 
20. Kim HK, Choi IJ, Kim CG, et al. A gene expression signature of acquired chemoresistance to cisplatin and fluorouracil combination chemotherapy in gastric cancer patients. PLoS One. 2011;6(2):e16694.

21. Erol O, Süren D, Tutuş B, et al. Immunohistochemical analysis of E-cadherin, p53 and inhibin- $\alpha$ expression in hydatidiform mole and hydropic abortion. Pathol Oncol Res. 2016;22(3):515-521.

22. Kwon SJ. Evaluation of the 7th UICC TNM Staging System of Gastric Cancer. J Gastric Cancer. 2011;11(2):78-85.
23. Spilka R, Ernst C, Mehta AK, Haybaeck J. Eukaryotic translation initiation factors in cancer development and progression. Cancer Lett. 2013;340(1):9-21.

24. Cuesta R, Gupta M, Schneider RJ. The regulation of protein synthesis in cancer. Prog Mol Biol Transl Sci. 2009;90:255-292.

25. Cheng Y, Zhou J, Li H. Clinicopathologic implications of eukaryotic initiation factor $3 \mathrm{f}$ and Her-2/neu expression in gastric cancer. Clin Transl Sci. 2015;8(4):320-325.

\section{Publish your work in this journal}

Cancer Management and Research is an international, peer-reviewed open access journal focusing on cancer research and the optimal use of preventative and integrated treatment interventions to achieve improved outcomes, enhanced survival and quality of life for the cancer patient. The manuscript management system is completely online and includes a very quick and fair peer-review system, which is all easy to use. Visit http://www.dovepress.com/testimonials.php to read real quotes from published authors. 